\title{
Thin-shell wormholes supported by ordinary matter in Einstein-Gauss-Bonnet gravity
}

\author{
Martín G. Richarte and Claudio Simeone* \\ Departamento de Física, Facultad de Ciencias Exactas y Naturales, Universidad de Buenos Aires \\ Ciudad Universitaria, Pabellón I, 1428, Buenos Aires, Argentina
}

December 14, 2018

\begin{abstract}
The generalized Darmois-Israel formalism for Einstein-Gauss-Bonnet theory is applied to construct thin-shell Lorentzian wormholes with spherical symmetry. We calculate the energy localized on the shell, and we find that for certain values of the parameters wormholes could be supported by matter not violating the energy conditions.
\end{abstract}

\section{Introduction}

For spacetime dimension $D \geq 5$ the Einstein-Hilbert action of gravity admits quadratic corrections constructed from coordinate-invariant tensors which scale as fourth derivatives of the metric. In particular, when $D=5$ the most general theory leading to second order equations for the metric is the so-called Einstein-Gauss-Bonnet theory or Lovelock theory up to second order. This class of model for higher dimensional gravity has been widely studied, in particular because it can be obtained in the low energy limit of string theory [1], and because it leads to ghost-free nontrivial gravitational self-interactions for $D>4$. For spacetime dimensions $D<5$ the Gauss-Bonnet terms in the action represent a topological invariant.

The equations of gravitation admit solutions, known as Lorentzian wormholes, which connect two regions of the same universe (or of two universes) by a throat, which is a minimal area surface [2, 3]. Such kind of geometries would present some features of particular interest, as for example the possibility of time travel (see Refs. [4). But a central objection against the actual existence of wormholes is that in Einstein gravity the flare-out condition [5] to be satisfied at the throat requires the presence of exotic matter, that is, matter violating the energy conditions 3 . In this sense, thin-shell wormholes have the advantage that the exotic matter would be located only at the shell.

However, it has recently been shown [6] that in pure Gauss-Bonnet gravity exotic matter is no needed for wormholes to exist; in fact, they could exist even with no matter (see also Ref. [7). In this work we thus study thin-shell wormholes in Einstein-Gauss-Bonnet gravity. We focus in the amount of matter necessary for supporting the wormholes, without analyzing the microphysics explaining this matter. Differing from the approach in the related work Ref. 8, where the Gauss-Bonnet

*E-mail: csimeone@df.uba.ar. 
terms were treated as an effective source for the Einstein's field equations, here the Gauss-Bonnet contribution is treated as an essentially geometrical object. This requires a generalization [9] of the Darmois-Israel formalism [10 for thin shells, but provides a better physical understanding. In particular, we show that for certain values of the parameters, thin-shell wormholes could be supported by matter not violating the energy conditions.

\section{Spherically symmetric geometry}

We start from the action for Einstein-Maxwell-Gauss-Bonnet theory in a five-dimensional manifold $\mathcal{M}_{5}$ with cosmological constant $\Lambda$ and Maxwell field [11]:

$$
S=\kappa \int_{\mathcal{M}_{5}} d^{5} x \sqrt{|g|}\left[R-2 \Lambda+\alpha R_{G B}^{2}-\frac{1}{4} F^{\mu \nu} F_{\mu \nu}\right],
$$

where $\kappa$ is related with the Newton constant, $R_{G B}^{2}=R^{2}-4 R^{a b} R_{a b}+R^{a b c d} R_{a b c d}$ is the GaussBonnet term, and $\alpha$ is the Gauss-Bonnet coupling constant. The Gauss-Bonnet constant introduces a scale $l_{G B} \propto \sqrt{\alpha}$ in the theory which physically represents a short-distance correction to general relativity. Within string theory, in five dimensions $\alpha$ would be of order the string mass scale; but in a more general framework $\alpha$ could be considered as an arbitrary real number with the appropriate dimensions.

The field equations resulting from the action (1) are

$$
G_{a b}+2 \alpha H_{a b}+\Lambda g_{a b}=\kappa^{2} T_{a b}
$$

where $H_{a b}$ is the second order Lovelock tensor and $T_{a b}$ is the usual electromagnetic energy-momentun tensor:

$$
\begin{gathered}
H_{a b}=R R_{a b}-2 R_{a c} R_{b}^{c}-2 R^{c d} R_{a c b d}+R_{a}^{c d e} R_{b c d e}-\frac{1}{4} g_{a b}\left(R^{2}-4 R^{c d} R_{c d}+R^{c d e q} R_{c d e q}\right), \\
T_{a b}=F_{a c} F_{b}^{c}-\frac{1}{4} g_{a b} F_{c d} F^{c d} .
\end{gathered}
$$

Equations (21) admit a spherically symmetric solution given by [11]:

$$
\begin{gathered}
d s^{2}=-f(r) d t^{2}+f^{-1}(r) d r^{2}+r^{2} d \Omega_{3}^{2}, \\
f(r)=1+\frac{r^{2}}{4 \alpha}\left[1-\sqrt{1+\frac{16 M \alpha}{\pi r^{4}}-\frac{8 Q^{2} \alpha}{3 r^{6}}+\frac{4 \Lambda \alpha}{3}}\right] .
\end{gathered}
$$

It is easy to check that in the limit $\alpha \rightarrow 0$ the five-dimensional Einstein-Maxwell solution with cosmological constant is recovered. Further for $\Lambda=0$ the five-dimensional Reissner-Nördstrom metric is obtained, so $M>0$ and $Q$ can be indentified with the mass and the charge of the system. For $\alpha \neq 0$, there is a minimun value of the radial coordinate $r_{\min }$ such that the function under the square root in (6) is positive so the metric (5) is well defined. The geometry has a curvature singularity at the surface defined by $r=r_{\text {min }}$. Depending on the values of the parameters $(M, \alpha, Q, \Lambda)$, this singular surface can be surrounded by an event horizon with a radius $r_{h o r}$, so the metric (5) represents a black hole; if no event horizont exists, it presents a naked singularity. Here 
we will focus in the case of null cosmological constant; the metric from which we start presents a singularity at $r_{\text {min }}$ given by the greatest real and positive solution of the equation

$$
r^{6}+\frac{16 M \alpha}{\pi} r^{2}-\frac{8 Q^{2} \alpha}{3}=0 .
$$

If Eq. (7) has no real positive solutions we have $r_{\min }=0$, where the metric diverges. This singularity is surrounded, in principle, by two horizons with radii

$$
r_{ \pm}=\sqrt{\frac{M}{\pi}-\alpha \pm\left[\left(\frac{M}{\pi}-\alpha\right)^{2}-\frac{Q^{2}}{3}\right]^{1 / 2}} .
$$

The event horizont is placed at $r_{h o r}=r_{+}$, and $r_{-}$corresponds to the inner horizon. If $\alpha>-M / \pi$ then $r_{\min }<r_{\text {hor }}$ and the singularity can be shielded by the event horizon. But if $\alpha \leq-M / \pi$, we have a naked singularity because $r_{\text {min }} \geq r_{h o r}$. It is not difficult to see that for $|\alpha|<M / \pi$, there exits a critical value of the charge:

$$
\left|Q_{c}\right|=\sqrt{3}\left|\frac{M}{\pi}-\alpha\right|
$$

such that if $|Q|<\left|Q_{c}\right|$ there are two horizons, if $|Q|=\left|Q_{c}\right|$ there is only one (degenerate) horizon, and if $|Q|>\left|Q_{c}\right|$ there are no horizons. For $|\alpha| \geq M / \pi$ no horizons exist for any value of the charge, except the nonphysical solution $r_{h o r}=0$ corresponding to $\alpha=M / \pi$ and $Q=0$.

\section{Thin-shell wormhole construction}

Starting from the metric given by (5) and (6) with $\Lambda=0$ we build a spherically symmetric thin-shell wormhole in the Einstein-Maxwell-Gauss-Bonnet theory. We take two copies of the spacetime and remove from each manifold the five-dimensional regions described by

$$
\mathcal{M}_{1,2}=\left\{r_{1,2} \leq b, b>r_{h o r}\right\} .
$$

The resulting manifolds have boundaries given by the timelike hypersurfaces

$$
\Sigma_{1,2}=\left\{r_{1,2}=b, b>r_{h o r}\right\} .
$$

Then we identify these two timelike hypersurfaces to obtain a geodesically complete new manifold $\mathcal{M}$ with a matter shell at the surface $r=b$, where the throat of the wormhole is located. This manifold is constituted by two asymptotically flat regions. To study this type of wormhole we apply the Darmois-Israel formalism generalized [9] to the case of Einstein- Gauss-Bonnet theory. We can introduce the coordinates $\xi^{i}=(\tau, \theta, \chi, \varphi)$ in $\Sigma$, with $\tau$ the proper time on the throat. Though we will focus mainly in static configurations, in the general case we could allow the radius of the throat be a function of the proper time, so that the boundary hypersurface reads:

$$
\Sigma: \mathcal{F}(r, \tau)=r-b(\tau)=0 .
$$

The field equations projected on the shell $\Sigma$ (generalized Darmois-Israel conditions) are 9]

$$
2\left[\left[K_{a b}-K h_{a b}\right]\right]+4 \alpha\left[\left[3 J_{a b}-J h_{a b}+2 P_{a b c d} K^{c d}\right]\right]=-\kappa^{2} S_{a b},
$$


where the double brakect stands for the jump of a given quantity across the hypersurface $\Sigma$. The extrinsic curvature $K_{a b}$, the divergence-free part of the Riemann tensor $P_{a b c d}$ and the tensor $J_{a b}$ are defined as follows:

$$
\begin{gathered}
K_{a b}^{ \pm}=-n_{c}^{ \pm}\left(\frac{\partial^{2} X^{c}}{\partial \xi^{a} \partial \xi^{b}}+\Gamma_{d e}^{c} \frac{\partial X^{d}}{\partial \xi^{a}} \frac{\partial X^{e}}{\partial \xi^{b}}\right)_{r=b} \\
P_{a b c d}=R_{a b c d}+\left(R_{b c} h_{d a}-R_{b d} h_{c a}\right)-\left(R_{a c} h_{d b}-R_{a d} h_{c b}\right)+\frac{1}{2} R\left(h_{a c} h_{d b}-h_{a d} h_{c b}\right) \\
J_{a b}=\frac{1}{3}\left[2 K K_{a c} K_{b}^{c}+K_{c d} K^{c d} K_{a b}-2 K_{a c} K^{c d} K_{d b}-K^{2} K_{a b}\right] .
\end{gathered}
$$

The tensor $P_{a b c d}$ is calculated with the induced metric $h_{a b}=g_{a b}-n_{a} n_{b}$ (see [9]). After some algebraic manipulation, the non-null components $S_{a}^{b}$ of the energy-momentun tensor of the shell are obtained as

$$
\begin{gathered}
S_{\tau}^{\tau}=\frac{1}{8 \pi}\left[6 \frac{\Delta}{b}+2 \alpha\left(24 \ell(b) \frac{\Delta}{b^{2}}+4 \frac{\Delta^{3}}{b^{3}}-12\left(1+\dot{b}^{2}\right) \frac{\Delta}{b^{3}}\right)\right], \\
S_{\theta}^{\theta}=S_{\varphi}^{\varphi}=S_{\chi}^{\chi}=\frac{1}{8 \pi}\left[4 \frac{\Delta}{b}+2 \ell(b) \frac{1}{\Delta}-2 \alpha\left(4 \ell(b) \frac{\Delta}{b^{2}}+4\left(1+\dot{b}^{2}\right) \frac{\ell(b)}{b^{2} \Delta}+4 \frac{\ddot{b} \Delta}{b}\right)\right],
\end{gathered}
$$

where $\ell(b)=\ddot{b}+f^{\prime}(b) / 2$ and $\Delta=\sqrt{\dot{b}^{2}+f(b)}$; the dot means a derivative with respect to the proper time and the prime with respect to $b$. From these equations we read the energy density $\sigma=-S_{\tau}^{\tau}$ and the tranverse pressure $p=S_{\theta}^{\theta}=S_{\chi}^{\chi}=S_{\varphi}^{\varphi}$ in terms of the throat radius $b(\tau)$, first and second derivatives of $b(\tau)$ and the function $f(b)$ wich depends on the parameters of the system. If we take $\alpha \rightarrow 0$ in both equations (17) and (18) we recover the expression for the energy density $\sigma$ and pressure $p$ found in Ref. 8 with the standard Lanczos equation for the shell. Furthermore, Taylor expanding up to zeroth order in $\alpha$ we recover the expressions for the five-dimensional Schwarzschild and Reissner-Nördstrom cases. Starting from Eqs. (17) and (18), in the next Section we will show that for certain values of the parameters, ordinary matter could support thin-shell wormholes in Einstein-Gauss-Bonnet theory.

\section{Matter supporting the wormholes; discussion}

Motivated by the results within pure Gauss-Bonnet gravity (i.e. without Einstein term) in Ref. [6], here we evaluate the amount of exotic matter and the energy conditions, following the approach presented above in which the Gauss-Bonnet term is treated as a geometrical contribution in the field equations. Coming this contribution from the curvature tensor, this approach is clearly the most suitable to give a precise meaning to the characterization of matter supporting the wormhole. As we shall see, the results will considerably differ from those in Ref. [8], where the Gauss-Bonnet term was treated as an effective source for the Einstein's field equations.

The weak energy condition (WEC) states that for any timelike vector $U^{\mu}$ it must be $T_{\mu \nu} U^{\mu} U^{\nu} \geq 0$; the WEC also implies, by continuity, the null energy condition (NEC), which means that for any null vector $k^{\mu}$ it mus be $T_{\mu \nu} k^{\mu} k^{\nu} \geq 0$ [3]. In an orthonormal basis the WEC reads $\rho \geq 0, \rho+p_{l} \geq 0 \forall l$, while the NEC takes the form $\rho+p_{l} \geq 0 \forall l$. In the case of thin-shell wormholes the radial pressure $p_{r}$ is zero, while within Einstein gravity or even with the inclusion of a Gauss-Bonnet term in the way proposed in [8], the surface energy density must fulfill $\sigma<0$, so that both energy conditions 
would be violated. The sign of $\sigma+p_{t}$ where $p_{t}$ is the transverse pressure is not fixed, but it depends on the values of the parameters of the system.

In what follows we restrict to static configurations. The surface energy density $\sigma_{0}$ and the transverse pressure $p_{0}$ for a static configuration $\left(b=b_{0}, \dot{b}=0, \ddot{b}=0\right)$ are given by

$$
\begin{gathered}
\sigma_{0}=-\frac{1}{8 \pi}\left[6 \frac{\sqrt{f\left(b_{0}\right)}}{b_{0}}+2 \alpha \sqrt{f\left(b_{0}\right)}\left(12 \frac{f^{\prime}\left(b_{0}\right)}{b_{0}^{2}}+4 \frac{f\left(b_{0}\right)}{b_{0}^{3}}-\frac{12}{b_{0}^{3}}\right)\right], \\
p_{0}=\frac{1}{8 \pi}\left[4 \frac{\sqrt{f\left(b_{0}\right)}}{b_{0}}+\frac{f^{\prime}\left(b_{0}\right)}{\sqrt{f\left(b_{0}\right)}}-2 \alpha\left(2 f^{\prime}\left(b_{0}\right) \frac{\sqrt{f\left(b_{0}\right)}}{b_{0}^{2}}+2 \frac{f^{\prime}\left(b_{0}\right)}{b_{0}^{2} \sqrt{f\left(b_{0}\right)}}\right)\right] .
\end{gathered}
$$

Note that the sign of the surface energy density is, in principle, not fixed. The most usual choice for quantifying the amount of exotic matter in a Lorentzian wormhole is the integral [12]:

$$
\Omega=\int\left(\rho+p_{r}\right) \sqrt{|g|} d^{4} x .
$$

We can introduce a new radial coordinate $\mathcal{R}= \pm\left(r-b_{0}\right)$ with \pm corresponding to each side of the shell. Then, because in our construction the energy density is located on the surface, we can write $\rho=\delta(\mathcal{R}) \sigma_{0}$, and because the shell does not exert radial pressure the amount of exotic matter reads

$$
\Omega=\int_{0}^{2 \pi} \int_{0}^{\pi} \int_{0}^{\pi} \int_{-\infty}^{+\infty} \delta(\mathcal{R}) \sigma_{0} \sqrt{|g|} d \mathcal{R} d \theta d \chi d \varphi=4 \pi^{2} b_{0}^{3} \sigma_{0} .
$$

Replacing the explicit form of $\sigma_{0}$ and $g$, we obtain the exotic matter amount as a function of the parameters that characterize the configurations:

$$
\Omega=-\frac{3}{2} \pi b_{0}^{2} \sqrt{f\left(b_{0}\right)}-2 \pi \alpha \sqrt{f\left(b_{0}\right)}\left[f\left(b_{0}\right)+3\left(b_{0} f^{\prime}\left(b_{0}\right)-1\right)\right],
$$

where $f$ is given by (6). For $\Lambda=0$, in the limit $\alpha \rightarrow 0$ and Taylor expanding up to zeroth order we obtain the exotic matter for the Reissner-Nördstrom $(Q \neq 0)$ and Schwarzschild $(Q=0)$ geometries.

For $\alpha>0$ we now find that there exist positive contributions to $\Omega$; these come from the different signs in the expression (19) for the surface energy density, because $\Omega$ is proportional to $\sigma_{0}$. We stress that this would not be possible if the standard Darmois-Israel formalism was applied, treating the Gauss-Bonnet contribution as an effective energy-momentum tensor, because this leads to $\sigma_{0} \sim$ $-\sqrt{f\left(b_{0}\right)} / b_{0}$ [8]. To simplify the analysis, without loss of physical interest, we consider the case $Q=0, \Lambda=0$. In figures 1 and 2 we show the would be amount of exotic matter as a function of the wormhole radius for relatively large values of $\alpha$ (that is, $\pi \alpha$ of order $M$ ); though this implies microscopic configurations or a scenario different from that suggested by present day observation, a numerical analysis shows that this is the most interesting situation. Besides the fact that $\Omega$ results to be smaller when calculated by treating the Gauss-Bonnet contribution as a geometric object than in the case that it was treated as an effective energy-momentum tensor, this amount is smaller than which would be necessary in the pure five-dimensional Schwazschild case (see Fig. 1). However, the central, remarkable, result is that now the existence of thin-shell wormholes is compatible with $\Omega>0$ (see Fig.2). Moreover, for these values of $\alpha$ and for $b$ about $\sqrt{M}$ (the horizon in the original manifold is at $r \approx \sqrt{M} / 4$ ), for which $\Omega>0$ and $\sigma_{0}>0$, we also have $p_{0}>0$, as it is easy to verify from Eq. (20). Thus, in the picture providing a clear meaning to matter in the shell, in EinsteinGauss-Bonnet gravity the violation of the energy conditions could be avoided, and wormholes could be supported by ordinary matter. 


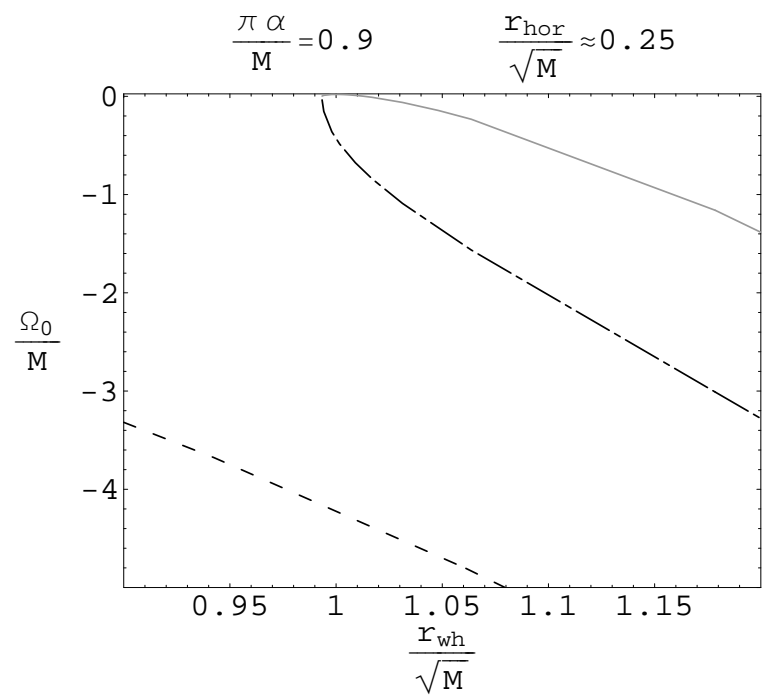

Figure 1: The amount $\Omega$ is shown as a function of $r_{w h} / \sqrt{M}$, for $Q=0$ and $\alpha=0.9$. The dasheddotted line corresponds to the five-dimensional Schwarzschild case, the dashed line corresponds to considering the Gauss-Bonnet term as a kind of effective source for the field equations, and the solid line shows the result obtained here with the generalized Darmois-Israel formalism for EinsteinGauss-Bonnet theory. The horizon radius corresponds to the Einstein-Gauss-Bonnet case.

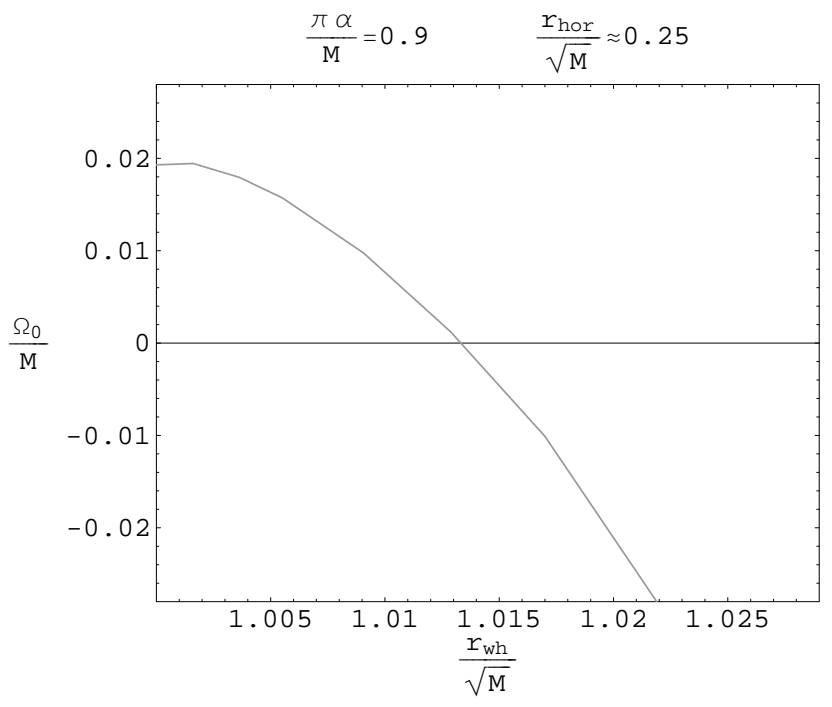

Figure 2: The amount $\Omega$ is shown as a function of $r_{w h} / \sqrt{M}$, for $Q=0$ and $\alpha=0.9$. The plot shows the result obtained here with the generalized Darmois-Israel formalism for Einstein-Gauss-Bonnet theory. The horizon radius corresponds to the Einstein-Gauss-Bonnet case. We see that we have the possibility of a thin-shell wormhole without violation of the energy conditions. 


\section{Acknowledgments}

We wish to thank M. Thibeault for important suggestions. This work was supported by Universidad de Buenos Aires and CONICET.

\section{References}

[1] B. Zwiebach, Phys.Lett. B 156315 (1985).

[2] M. S. Morris and K. S. Thorne, Am. J. Phys. 56, 395 (1988).

[3] M. Visser, Lorentzian Wormholes (AIP Press, New York, 1996).

[4] M. S. Morris, K. S. Thorne and U. Yurtsever, Phys. Rev. Lett 61, 1446 (1988); V. P. Frolov and I. D. Novikov, Phys. Rev. D 42, 1057 (1990).

[5] D. Hochberg and M. Visser, Phys. Rev. D 56, 4745 (1997).

[6] E. Gravanis and S. Willison, Phys. Rev. D 75, 084025 (2007).

[7] G. Dotti, J. Oliva and R. Troncoso, Phys. Rev. D 75, 024002 (2007).

[8] M. Thibeault, C. Simeone and E. F. Eiroa, Gen. Relativ. Gravit. 38, 1593 (2006).

[9] S. C. Davis, Phys. Rev. D 67, 024030 (2003).

[10] N. Sen, Ann. Phys. (Leipzig) 73, 365 (1924); K. Lanczos, ibid. 74, 518 (1924); G. Darmois, Mémorial des Sciences Mathématiques, Fascicule XXV ch V (Gauthier-Villars, Paris, 1927); W. Israel, Nuovo Cimento 44B, 1 (1966); 48B, 463(E) (1967).

[11] D. L. Wiltshire, Phys. Rev D 382445 (1988).

[12] K. K. Nandi, Y.-Z. Zhang, and K. B. Vijaya Kumar, Phys. Rev. D 70, 127503 (2004); K. K. Nandi, Y.-Z. Zhang and N. G. Migranov, J. Nonlinear Phenomena in Complex Systems 9, 61 (2006) (arXiv:gr-qc/0409053); F. S. N. Lobo, Gen. Relativ. Gravit. 37, 2023 (2005); E. F. Eiroa and C. Simeone, Phys. Rev. D 71, 127501 (2005). 